\title{
BMJ Open Tai Chi and other mind-body interventions for cancer-related fatigue: an updated systematic review and network meta-analyses protocol
}

\author{
Hong-Juan Fu (D) ${ }^{1,2}$ Hao Zhou, ${ }^{2}$ Yong Tang, ${ }^{3}$ Jie Li, ${ }^{1}$ Da Zhang, ${ }^{2}$ Song-Yi Ding, ${ }^{2}$ \\ Qin-Wan Huang, ${ }^{4}$ Chao Wang ${ }^{1,2}$
}

To cite: Fu H-J, Zhou H, Tang Y, et al. Tai Chi and other mind-body interventions for cancer-related fatigue: an updated systematic review and network metaanalyses protocol. BMJ Open 2022;12:e052137. doi:10.1136/ bmjopen-2021-052137

- Prepublication history and additional supplemental material for this paper are available online. To view these files, please visit the journal online (http://dx.doi.org/10.1136/ bmjopen-2021-052137).

Received 08 April 2021 Accepted 21 December 2021

Check for updates

(c) Author(s) (or their employer(s)) 2022. Re-use permitted under CC BY-NC. No commercial re-use. See rights and permissions. Published by BMJ.

${ }^{1}$ Acupuncture and Tuina School, Chengdu University of Traditional Chinese Medicine Shierqiao Campus, Chengdu, China

${ }^{2}$ Sichuan Integrative Medicine Hospital, Chengdu, China

${ }^{3}$ Acupuncture-Moxibustion School, Chengdu University of Traditional Chinese Medicine Affiliated Hospital, Chengdu, China

${ }^{4}$ School of Pharmacy, Chengdu University of Traditional Chinese Medicine, Chengdu, China

Correspondence to

Dr Chao Wang;

wc15520776001@163.com

\section{ABSTRACT}

Introduction Fatigue is one of the most common symptoms in patients with cancer and is responsible for a reduced quality of life. There is a strong evidence base for mind-body interventions (MBIs) to manage cancer-related fatigue (CRF). However, the efficacy of Tai Chi and other MBIs in the treatment of CRF remains controversial. Methods and analysis We will perform a systematic review and network meta-analyses (NMAs) that aim to assess the effects of Tai Chi and other MBIs in patients with CRF. The following databases will be searched from their inception to 1 August 2021: PubMed, EMBASE, Scopus, OVID, Web of Science, Cochrane Central Register of Controlled Trials, the China National Knowledge Infrastructure, China Science and Technology Journal Database, Chinese Biomedical Database and Wan Fang Digital Journals. We will include randomised controlled trials that compare MBIs with no treatment, placebo and usual care in the treatment of CRF. The primary outcome will be changes in the fatigue state as evaluated by validated scales. We will perform a Bayesian NMA to analyse all the evidence for each outcome. The surface under the cumulative ranking curve and the mean ranks will be used to rank the various treatments. We will assess the quality of evidence contributing to network estimates of outcomes using the Grading of Recommendations Assessment, Development and Evaluation system framework.

Ethics and dissemination This NMAs will be disseminated through publication in a peer-reviewed journal. Since no individual patient data will be involved in the review, ethics approval and concerns about privacy are not needed.

PROSPERO registration number CRD42021244999.

\section{INTRODUCTION}

Cancer-related fatigue (CRF) is defined as 'a distressing, persistent, subjective sense of physical, emotional, and/or cognitive tiredness or exhaustion related to cancer or cancer treatment that is not proportional to recent activity and which interferes with usual functioning' by the National Comprehensive Cancer Network (NCCN). ${ }^{1}$ CRF is
Strengths and limitations of this study

- This study will include the latest randomised controlled trials to update the evidence base and obtain a comprehensive ranking of all included treatments.

- The revised Cochrane risk of bias tool will be used to assess the risk of bias in eligible studies.

- Grading of Recommendations Assessment, Development and Evaluation system will be used to assess the quality of evidence.

- Different types of mind-body interventions (MBIs) may cause considerable heterogeneity in this review.

- Some MBIs, such as acupuncture and massage, will not be included in this review; this may affect the results.

one of the most prevalent and distressing symptoms of cancer, and it might persist for years after treatment completion in survivors. ${ }^{2}{ }^{3}$ CRF estimates range from $14.03 \%$ to $100 \%$ depending on the latest research. ${ }^{45}$ Furthermore, CRF has a significant effect on physical functioning during treatment, and it is uncertain whether patients regain full functioning after completion of treatment. Persistent CRF causes disruption in all aspects of quality of life (QoL) during and after treatment. ${ }^{67}$ Furthermore, CRF can cause difficulties in end-of-life care and it might be a risk factor for reduced survival. ${ }^{1}$

The pathophysiology of CRF remains unknown. The proposed underlying CRF mechanisms include mitochondrial dysfunction, peripheral immune activation, inflammation dysfunction and central mechanisms (neuropeptide, neurotransmitter, hypothalamic-pituitary-adrenal axis dysfunction). ${ }^{89}$ To the best of our knowledge, there is no gold standard for the management of CRF. Based on the NCCN for CRF, some nonpharmacologic interventions have shown a strong evidence base for treating CRF. ${ }^{1}$ 
Interestingly, there is an increasing interest in mindbody interventions (MBIs), such as Tai Chi, in oncology settings, which may provide a new and effective treatment for CRF. ${ }^{10}$

The National Center for Complementary and Integrative Health defined MBIs as 'techniques designed to enhance the mind's capacity to affect bodily function and symptoms'. ${ }^{11}$ MBIs have been shown to be effective in decreasing the expression of inflammation-related genes and in reducing common cancer-related side effects, especially in alleviating fatigue symptoms. ${ }^{12-14}$ Tai Chi is rooted in traditional Chinese medicine and has been practiced for several millennia. This complex, multicomponent MBI merges physical, spiritual, psychosocial and behavioural elements to promote human health. ${ }^{15}$ Randomised controlled trials (RCTs) have indicated that Tai Chi significantly alleviates fatigue in patients with lung cancer and breast cancer over time. ${ }^{1617}$

However, the effect of Tai Chi on CRF is still controversial based on evidence from several systematic reviews and meta-analyses. ${ }^{18-20}$ The inconsistent results may be due to differences in search strategies, inclusion criteria and comparators in these reviews. Furthermore, published meta-analyses only reviewed trials before 2016. Recently, new RCTs have been conducted and published. ${ }^{21-23}$ To provide comprehensive evidence for the treatment of CRF, it is necessary to re-evaluate the effectiveness of Tai Chi and other MBIs for CRF based on the latest resources available.

\section{Objectives}

We aimed to collect RCTs comparing Tai Chi and other MBIs with placebo or other non-MBIs among patients with CRF, and to conduct network meta-analyses (NMAs) to assess the comparative effects of Tai Chi and other MBIs on CRF.

\section{METHODS AND ANALYSIS}

The study will be conducted according to the Preferred Reporting Items for Systematic Reviews and Meta-Analyses (PRISMA) for systematic review protocols ${ }^{24}$ (http://www. prisma-statement.org/Extensions/Protocols.aspx) and NMA checklist ${ }^{25}$ (http://www.prisma-statement.org/ Extensions/NetworkMetaAnalysis.aspx). This review protocol has been registered in PROSPERO.

\section{Criteria for consideration of studies in this review \\ Types of studies}

RCTs on Tai Chi and other MBIs for CRF with fatigue outcomes will be included in this review. No limits will be applied to the language, publication status and publication date of these studies.

\section{Types of participants}

Patients aged 18 years or older, of both sexes, with a diagnosis of CRF will be considered. No restrictions will be set for cancer type, cancer grade, during treatment or after end of treatment in patients.

Types of interventions

We focus here on four types of MBIs that have received considerable research attention and are widely available to clinical and community populations: Tai Chi (such as Yang-style Tai Chi, Chen-style Tai Chi, Wu-style Tai Chi, Sun-style Tai Chi, 24 simplified Tai Chi or movements of Tai Chi), Qi gong (including Baduanjin, Yijinjing and Wuqinxi), yoga and meditation. In this study, all different types of Tai Chi and Qi gong will first be reviewed as the same intervention and compared with other control measures. Then, different types of Tai Chi and Qi gong will be analysed again as different interventions.

\section{Types of comparators}

To determine whether the effects of Tai Chi and Qi gong are primarily due to physical activity and whether the effects of yoga are primarily due to stretching, exercise and stretching will be included as a control group.

We included and classified the comparators in studies as follows:

1. Tai Chi and other MBIs versus exercise/stretching/ placebo therapies.

2. Tai Chi and other MBIs versus waiting list/no treatment/usual care.

3. MBIs versus MBIs.

Types of outcome measures

\section{Primary outcome}

We will extract the effect sizes at the first time point after the end of the interventions and the subsequent follow-up time points. Only RCTs that include the following primary outcomes, namely, assessments of CRF using effective and validated scales such as Brief Fatigue Inventory, Multidimensional Fatigue Symptom Inventory-Short Form, Functional Assessment of Chronic Illness Therapy-Fatigue Survey and Functional Assessment of Cancer TherapyFatigue, will be included in this review.

\section{Secondary outcomes}

The secondary outcomes will include QoL and adverse events. Questionnaires will be used to assess QoL. Questionnaires will be used for assessment of QoL, such as the Functional Assessment of Cancer Therapy, European Organisation for Research and Treatment of Cancer Quality of Life Questionnaire Core 30, Quality of Life Questionnaire Breast Cancer Module 23, 36-Item Short Form Health Survey, Quality of Life in Adult Cancer Survivors and WHO Quality of Life Questionnaire.

\section{Search methods}

Published RCTs will be searched in the following electronic databases: PubMed, EMBASE, Scopus, OVID, Web of Science, Cochrane Central Register of Controlled Trials, the China National Knowledge Infrastructure, China Science and Technology Journal Database, Chinese Biomedical Databases and Wan Fang Digital Journals 
from the date of their inception to 1 August 2021 with no restrictions on language. Unpublished trials will be retrieved from the following clinical trial registries: the NIH clinical registry (ClinicalTrials.gov), Australian New Zealand Clinical Trials Registry and Chinese Clinical Registry. In addition, the reference lists of all relevant articles will be checked to identify additional studies.

The medical search headings (MeSH) terms and their synonyms (free text) will be combined using the Boolean operators: 'AND' and 'OR'. The following MeSH terms will be used: 'cancer', 'tumor', 'carcinoma', 'neoplasm', 'fatigue', 'Tai Chi', 'Qi gong', 'Yoga', 'meditation' and 'randomized controlled trial'. The search strategies are shown in online supplemental file.

\section{Data collection and analysis}

\section{Selection of studies}

Two researchers (HF and HZ) will independently select studies using the bibliographic software EndNote (https://www.endnote.com/). Initially, duplicate studies will be filtered out. The researchers will then read the titles and abstracts to exclude studies that do not satisfy the eligibility criteria. After this selection, the full text of all remaining articles will be extracted to determine which articles can be included. In the case of duplicate studies, we will include only those trials with the most informative data. A third researcher ( $\mathrm{Q}-\mathrm{WH})$ is responsible for reaching a consensus with the two authors on the inclusion or exclusion of each study. Finally, we will provide a list of excluded studies and justify the exclusions. The selection procedure will be shown in a PRISMA flow chart (figure 1).

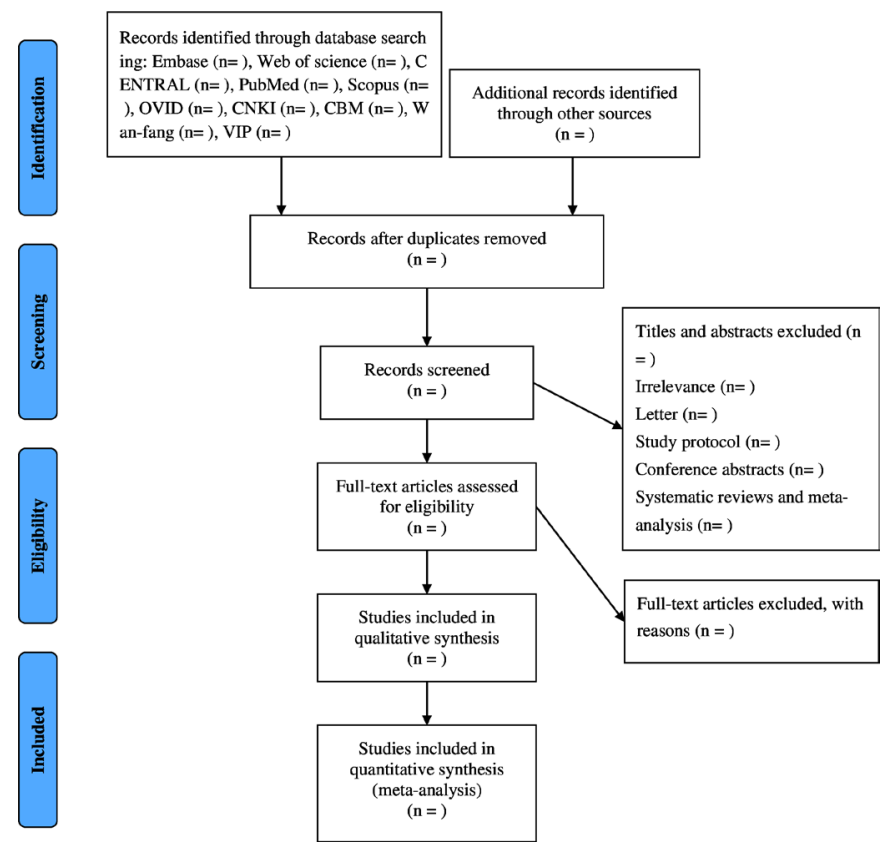

Figure 1 Flow chart of the study. CBM, Chinese Biomedical; CENTRAL, Cochrane Central Register of Controlled Trials; CNKI, China National Knowledge Infrastructure; VIP, the Chongqing VIP Chinese Science and Technology Periodical DatabaseApprove.

\section{Data extraction}

Two researchers (YT and DZ) will independently extract data using ADDIS (http://www.drugis.org/index) and Excel software with respect to five main domains: study information (eg, title, source of publication, year of publication, first author's name and affiliation, and country), participant information (eg, gender, age, setting, cancer type, tumour grade and basic cancer treatment plan), intervention details (eg, intervention type, duration and frequency), methodology information (study design, random sequence generation, allocation concealment, blinding and other concerns about bias) and outcome measures. The two researchers will cross-check and ascertain data accuracy. Disagreements will be resolved by a third researcher (S-YD).

\section{Unit of analysis issues}

This is not an individual participant data review, and all analyses will be based on aggregated outcome data from the included RCTs.

\section{Dealing with missing data}

We will send a request for missing data to the original investigators of the trial or the contact person recorded in the trial registry. In case of no reply, we will use the last observation carried forward imputation method and impute the missing data with replacement values (https:/ / training.cochrane.org/handbook/current/chapter-06). If means, SDs and numbers of patients in each arm are not reported, we will transform the recorded SEs, t statistics or $p$ values to SDs, according to the Cochrane Handbook. ${ }^{26}{ }^{27}$ If possible, we will perform sensitivity analyses to assess how sensitive the results are to reasonable changes in the assumptions that are made. The potential impact of missing data on the findings of the review will be addressed in the Discussion section.

\section{Quality assessment}

Two independent researchers (YT and JL) will assess the risk of bias of the included RCTs in accordance with the revised Cochrane risk of bias tool. ${ }^{28-30}$ Each domain will be rated as low risk, some concern and high risk, based on the five distinct domains: the randomisation process, deviations from the intended interventions, missing outcome data, measurement of the outcome and selection of the reported result. We will also make an overall risk of bias judgement according to the following criteria $^{31}$ : (1) low risk-all domains for this result; (2) some concerns-in at least one domain for this result but not at high risk of bias for any domain; (3) high risk-in at least one domain for this result, or the study is judged to have some concerns for multiple domains in a way that substantially lowers confidence in the results. If the researchers disagree, a third researcher (CW) will resolve the differences.

\section{Data synthesis and analysis}

The evidence-base and information flow in the network

We will systematically and comprehensively describe the characteristics of all eligible trials. A network diagram will 
be used to present the available evidence. ${ }^{32}$ The size of the nodes will represent the sample size of the included trials. The thickness of the line will reflect the number of studies for each direct comparison, and the colour of each edge will imply the risk of bias. For NMAs, including many competing interventions and multi-arm studies, we will instead use a table to show the network structure. ${ }^{33} 34$ To understand how much each of the direct comparisons contributes to the final summary data, the contribution matrix will be used to display the percentage information of the direct evidence contributing to each relative effect estimated for a study. ${ }^{35}$

\section{Pairwise meta-analyses}

We will use the DerSimonian-Laird random effects model for pairwise comparison. Standardised mean differences (SMD) for continuous outcomes or ORs for dichotomous outcomes, both with a $95 \% \mathrm{CI}$, will be calculated as effect measures. The Cochran's $Q \chi^{2}$ test and $\mathrm{I}^{2}$ statistic will be measured for heterogeneity as a measure to reflect the underlying differences between the RCTs that directly compare the same pair of interventions. A $p$ value of up to 0.10 , and $\mathrm{I}^{2}$ value of above $50 \%$ will indicate high heterogeneity ${ }^{29}$ We will perform sensitivity analysis of pairwise meta-analyses to validate the robustness of the results by omitting studies with unacceptable sources of heterogeneity.

\section{Examination of assumptions in NMAs (transitivity, inconsistency} and heterogeneity)

Studies that compare different interventions may differ in a broad range of characteristics, which are sometimes associated with the effect of an intervention and are referred to as effect modifiers. We will evaluate the transitivity assumption underlying NMAs by comparing the distribution of clinical and methodological variables, which can act as effect modifiers across treatment comparisons. ${ }^{262736}$ Consistency in NMA means that the different sources of evidence (direct and indirect) are consistent with each other. We will employ the node splitting method and heatmap to investigate the inconsistency of the model by separating evidence on a particular comparison into direct and indirect evidence. ${ }^{27} 36$ The loopspecific approach will be used to evaluate the presence of inconsistency locally in each closed loop. ${ }^{27}{ }^{36}$ We will also calculate the $\mathrm{I}^{2}$ statistic to evaluate consistency and heterogeneity in the entire network. ${ }^{276}$ If there is inconsistency and considerable heterogeneity in the RCTs, NMAs will not be performed and a narrative systematic review will be provided instead.

\section{Network meta-analyses}

All analyses will be performed using the gemtc, netmeta and ggplot package of R V.3.5.0 and the network package in Stata V.15.1. If the included trials meet the above three assumptions, we will perform a random-effects NMA within a Bayesian framework. We will fit our model using WinBUGS (V.1.4, 3 Markov chains, 50000 iterations, an initial burn-in of 10000 and a thinning of 10) and use uninformative prior distributions for the treatment effects. The binomial likelihood will be used for dichotomous outcomes and the normal likelihood for continuous outcomes. ${ }^{26}{ }^{27}$ ORs or SMD for all pairwise comparisons with $95 \%$ CI will be summarised in a league table. We will use the surface under the cumulative ranking curve (SUCRA) and the mean ranks to rank the various interventions for all outcomes. ${ }^{38}$ A SUCRA equal to 1 means that the treatment is considered to be the best, while 0 means that it is certain to be the worst.

\section{Publication bias, subgroup analyses and meta-regression}

We will perform a contribution plot to evaluate the contribution of each direct comparison to the assessment of each network meta-analytic summary effect. Additionally, we will use comparison-adjusted funnel plots to detect the potential publication bias in the results between imprecise and more precise trials. ${ }^{39}$ To assess whether the treatment effects for the primary outcome are impacted by effect modifiers, subgroup analyses and network meta-regression will be conducted according to the following characteristics: (1) cancer type, (2) patient status (ongoing or post cancer treatment or no treatment), (3) randomisation and blinding and (4) sample size (fewer than 25 patients per intervention arm). The sensitivity analysis of NMA will be narrowed into head-tohead studies or trials with a low risk of bias.

\section{Summary of evidence}

Two experienced researchers (HZ and DZ) will independently assess the certainty of evidence contributing to each network estimate of the primary outcome using the Grading of Recommendations Assessment, Development and Evaluation framework. Evidence quality will be rated as high, moderate, low or very low on the basis of the study limitations, imprecision, inconsistency, indirectness and publication bias. ${ }^{40}$ Discrepancies will be resolved by a third researcher $(\mathrm{CW})$.

\section{Patient and public involvement}

This review will not recruit patients, and they will not directly be involved in the design and implementation of this study.

\section{Ethics and dissemination}

This NMAs will be disseminated through publication in a peer-reviewed journal. Since no individual patient data will be involved in the review, ethics approval and concerns about privacy are not needed.

\section{DISCUSSION}

Although the effect of Tai Chi on CRF is still controversial, Tai Chi has the potential to be an effective complementary treatment option for CRF. In this review, we will perform the most comprehensive and up-to-date literature search, including 10 electronic databases and clinical research registration websites. We will also extract 
the effect sizes at the end of the intervention period and post-intervention follow-up to evaluate the shortterm and long-term efficacy. Pairwise and NMAs will also be performed. We are confident that this research can update the evidence of Tai Chi and other MBIs in the treatment of CRF and provide evidence-based medicine for patients, clinicians and policy-makers.

Acknowledgements We would like to thank Editage (www.editage.com) for English language editing.

Contributors HF and HZ contributed equally. HF, YT and CW conceived the review protocol. HF drafted the manuscript. Q-WH and CW revised the study design. HF, $\mathrm{HZ}$ and $\mathrm{Q}-\mathrm{WH}$ will perform the study search and study selection. YT, DZ and S-YD will carry out the data collection. YT, JL and CW will assess the quality of included randomised controlled trials. HF and YT will conduct the data analysis. Q-WH and CW will monitor each procedure of the review and are responsible for the quality control. All authors have read and approved the publication of the protocol.

Funding This study was supported by grants from the Sichuan Province Traditional Chinese Medicine Academician Reserve Candidate Training Program, the Crosssectional Study on Correlation Between Constitutional Type of Sub-health Insomnia Patients and Acupoint Sensitization, and the Research and Development of Health Food "Anshen-kangjuan Biscuits" (Grant No.2021MS014).

Disclaimer The sponsors had no role in developing the protocol.

Competing interests None declared.

Patient consent for publication Not applicable.

Provenance and peer review Not commissioned; externally peer reviewed.

Data availability statement No additional data available.

Supplemental material This content has been supplied by the author(s). It has not been vetted by BMJ Publishing Group Limited (BMJ) and may not have been peer-reviewed. Any opinions or recommendations discussed are solely those of the author(s) and are not endorsed by BMJ. BMJ disclaims all liability and responsibility arising from any reliance placed on the content. Where the content includes any translated material, BMJ does not warrant the accuracy and reliability of the translations (including but not limited to local regulations, clinical guidelines, terminology, drug names and drug dosages), and is not responsible for any error and/or omissions arising from translation and adaptation or otherwise.

Open access This is an open access article distributed in accordance with the Creative Commons Attribution Non Commercial (CC BY-NC 4.0) license, which permits others to distribute, remix, adapt, build upon this work non-commercially, and license their derivative works on different terms, provided the original work is properly cited, appropriate credit is given, any changes made indicated, and the use is non-commercial. See: http://creativecommons.org/licenses/by-nc/4.0/.

\section{ORCID iD}

Hong-Juan Fu http://orcid.org/0000-0003-1211-3814

\section{REFERENCES}

1 Berger AM, Mooney K, Alvarez-Perez A, et al. Cancer-Related fatigue, version 2.2015. J Natl Compr Canc Netw 2015;13:1012-39.

2 Ahlberg K, Ekman T, Gaston-Johansson F, et al. Assessment and management of cancer-related fatigue in adults. Lancet 2003;362:640-50.

3 Bower JE. Cancer-related fatigue--mechanisms, risk factors, and treatments. Nat Rev Clin Oncol 2014;11:597-609.

4 Strebkova R. Cancer-Related fatigue in patients with oncological diseases: causes, prevalence, guidelines for assessment and management. Folia Med 2020;62:679-89.

$5 \mathrm{Ma} \mathrm{Y}, \mathrm{He} \mathrm{B}$, Jiang M, et al. Prevalence and risk factors of cancerrelated fatigue: a systematic review and meta-analysis. Int $J$ Nurs Stud 2020;111:103707.

6 Charalambous A, Kouta C. Cancer related fatigue and quality of life in patients with advanced prostate cancer undergoing chemotherapy. Biomed Res Int 2016;2016:3989286

7 Iwase S, Kawaguchi T, Tokoro A, et al. Assessment of cancer-related fatigue, pain, and quality of life in cancer patients at palliative care team referral: a multicenter observational study (JORTC PAL-09). PLoS One 2015;10:e0134022.
8 Yang S, Chu S, Gao Y, et al. A narrative review of cancer-related fatigue (CRF) and its possible pathogenesis. Cells 2019;8. doi:10.3390/cells8070738. [Epub ahead of print: 1807 2019].

9 O'Higgins CM, Brady B, O'Connor B, et al. The pathophysiology of cancer-related fatigue: current controversies. Support Care Cancer 2018;26:3353-64.

10 Lopez G, Narayanan S, Christie A, et al. Effects of Center-Based delivery of tai chi and Qi Gong group classes on self-reported symptoms in cancer patients and caregivers. Integr Cancer Ther 2020;19:1534735420941605.

11 Bremner M, Blake B, Stiles C. The experiences of persons living with HIV who participate in Mind-body and energy therapies: a systematic review protocol of qualitative evidence. JBI Database System Rev Implement Rep 2015;13:41-9.

12 Duan L, Xu Y, Li M. Effects of Mind-body exercise in cancer survivors: a systematic review and meta-analysis. Evid Based Complement Alternat Med 2020;2020:1-13.

13 Hall DL, Luberto CM, Philpotts LL, et al. Mind-Body interventions for fear of cancer recurrence: a systematic review and meta-analysis. Psychooncology 2018;27:2546-58.

14 Carlson LE, Zelinski E, Toivonen K, et al. Mind-Body therapies in cancer: what is the latest evidence? Curr Oncol Rep 2017;19:67.

15 Wang C, Schmid CH, Fielding RA, et al. Effect of tai chi versus aerobic exercise for fibromyalgia: comparative effectiveness randomized controlled trial. BMJ 2018;360:k851.

16 Zhang L-L, Wang S-Z, Chen H-L, et al. Tai chi exercise for cancer-related fatigue in patients with lung cancer undergoing chemotherapy: a randomized controlled trial. J Pain Symptom Manage 2016;51:504-11.

17 Larkey LK, Roe DJ, Weihs KL, et al. Randomized controlled trial of Qigong/Tai chi easy on cancer-related fatigue in breast cancer survivors. Ann Behav Med 2015;49:165-76.

18 Song S, Yu J, Ruan Y, et al. Ameliorative effects of tai chi on cancerrelated fatigue: a meta-analysis of randomized controlled trials. Support Care Cancer 2018;26:2091-102.

19 Hilfiker R, Meichtry A, Eicher M, et al. Exercise and other nonpharmaceutical interventions for cancer-related fatigue in patients during or after cancer treatment: a systematic review incorporating an indirect-comparisons meta-analysis. $\mathrm{Br} J$ Sports Med 2018;52:651-8

20 Xiang Y, Lu L, Chen X, et al. Does tai chi relieve fatigue? A systematic review and meta-analysis of randomized controlled trials. PLoS One 2017;12:e0174872.

21 Zhou W, Wan Y-H, Chen Q, et al. Effects of tai chi exercise on cancer-related fatigue in patients with nasopharyngeal carcinoma undergoing chemoradiotherapy: a randomized controlled trial. J Pain Symptom Manage 2018;55:737-44.

22 McQuade JL, Prinsloo S, Chang DZ, et al. Qigong/tai chi for sleep and fatigue in prostate cancer patients undergoing radiotherapy: a randomized controlled trial. Psychooncology 2017;26:1936-43.

23 Irwin MR, Olmstead R, Carrillo C, et al. Tai chi Chih compared with cognitive behavioral therapy for the treatment of insomnia in survivors of breast cancer: a randomized, partially blinded, Noninferiority trial. J Clin Oncol 2017;35:2656-65.

24 Moher D, Shamseer L, Clarke M, et al. Preferred reporting items for systematic review and meta-analysis protocols (PRISMA-P) 2015 statement. Syst Rev 2015;4:1.

25 Hutton B, Salanti G, Caldwell DM, et al. The PRISMA extension statement for reporting of systematic reviews incorporating network meta-analyses of health care interventions: checklist and explanations. Ann Intern Med 2015;162:777-84.

26 Furukawa TA, Salanti G, Atkinson LZ, et al. Comparative efficacy and acceptability of first-generation and second-generation antidepressants in the acute treatment of major depression: protocol for a network meta-analysis. BMJ Open 2016;6:e010919.

27 Cipriani A, Furukawa TA, Salanti G, et al. Comparative efficacy and acceptability of 21 antidepressant drugs for the acute treatment of adults with major depressive disorder: a systematic review and network meta-analysis. Lancet 2018;391:1357-66.

28 Siemieniuk RAC, Bartoszko JJ, Ge L, et al. Drug treatments for covid-19: living systematic review and network meta-analysis. BMJ 2020;45:m2980.

29 Chiarito M, Sanz-Sánchez J, Cannata F, et al. Monotherapy with a P2Y inhibitor or aspirin for secondary prevention in patients with established atherosclerosis: a systematic review and meta-analysis. Lancet 2020;395:1487-95.

30 Lopes-Júnior LC, Bomfim E, Olson K, et al. Effectiveness of hospital clowns for symptom management in paediatrics: systematic review of randomised and non-randomised controlled trials. BMJ 2020;371:m4290. 
31 Sterne JAC, Savović J, Page MJ, et al. Rob 2: a revised tool for assessing risk of bias in randomised trials. BMJ 2019;366:14898.

32 Chaimani A, Higgins JPT, Mavridis D, et al. Graphical tools for network meta-analysis in STATA. PLoS One 2013;8:e76654.

33 Baker WL, Baker EL, Coleman Cl. Pharmacologic treatments for chronic obstructive pulmonary disease: a mixed-treatment comparison meta-analysis. Pharmacotherapy 2009;29:891-905.

34 Chen AM, Bollmeier SG, Finnegan PM. Long-Acting bronchodilator therapy for the treatment of chronic obstructive pulmonary disease. Ann Pharmacother 2008;42:1832-42.

35 Chaimani A, Salanti G. Visualizing assumptions and results in network meta-analysis: the network graphs package. Stata $J$ 2015;15:905-50.

36 Wu S, Gao L, Cipriani A, et al. The effects of incretin-based therapies on $\beta$-cell function and insulin resistance in type 2 diabetes: a systematic review and network meta-analysis combining 360 trials. Diabetes Obes Metab 2019;21:975-83.

37 Dias S, Welton NJ, Caldwell DM, et al. Checking consistency in mixed treatment comparison meta-analysis. Stat Med 2010;29:932-44.

38 Salanti G, Ades AE, loannidis JPA. Graphical methods and numerical summaries for presenting results from multiple-treatment metaanalysis: an overview and tutorial. J Clin Epidemiol 2011;64:163-71.

39 Furukawa TA, Miura T, Chaimani A, et al. Using the contribution matrix to evaluate complex study limitations in a network metaanalysis: a case study of bipolar maintenance pharmacotherapy review. BMC Res Notes 2016;9:218.

40 Salanti G, Del Giovane C, Chaimani A, et al. Evaluating the quality of evidence from a network meta-analysis. PLoS One 2014;9:e99682. 